\title{
Fine-Grained Access Control with Object-Sensitive Roles ${ }^{\star}$
}

\author{
Jeffrey Fischer, Daniel Marino, Rupak Majumdar, and Todd Millstein \\ Computer Science Department \\ University of California, Los Angeles \\ \{fischer, dlmarino, rupak, todd $\}$ ecs.ucla.edu
}

\begin{abstract}
Role-based access control (RBAC) is a common paradigm to ensure that users have sufficient rights to perform various system operations. In many cases though, traditional RBAC does not easily express application-level security requirements. For instance, in a medical records system it is difficult to express that doctors should only update the records of their own patients. Further, traditional RBAC frameworks like Java's Enterprise Edition rely solely on dynamic checks, which makes application code fragile and difficult to ensure correct.

We introduce Object-sensitive RBAC (ORBAC), a generalized RBAC model for object-oriented languages. ORBAC resolves the expressiveness limitations of RBAC by allowing roles to be parameterized by properties of the business objects being manipulated. We formalize and prove sound a dependent type system that statically validates a program's conformance to an ORBAC policy. We have implemented our type system for Java and have used it to validate fine-grained access control in the OpenMRS medical records system.
\end{abstract}

\section{Introduction}

Controlled access to data and operations is a key ingredient of system security. Rolebased access control (RBAC) [9] is an elegant and frequently-used access control mechanism in which a layer of roles interposes between users and access privileges. Roles represent responsibilities within a given organization. Authorizations for resource access are granted to roles rather than to individual users and users are given roles according to their functions in the organization. Users acquire all privileges associated with their roles. The intuition behind RBAC is that roles change infrequently within organizations relative to users, and so associating roles with access privileges ensures a stable and reliable access control policy.

As a concrete scenario, consider a hospital in which users can be doctors or patients. Doctors should be able to view and update their patients' records, and patients should be able to view (but not update) their own records. The RBAC way to represent this policy is to introduce two roles Doctor and Patient, where the Doctor role is allowed to both look up and modify patient records and the Patient role is allowed only to look up a medical record. Users are then classified as having the Doctor or Patient roles and

\footnotetext{
* This material is based upon work supported in part by the National Science Foundation under grants CCF-0545850 and CCF-0546170.
} 
inherit the corresponding access privileges. RBAC is available in standard enterprise software development environments such as Java's Enterprise Edition (Java EE) [16], which insert runtime role checks whenever a privileged operation is invoked.

This simple example highlights two key limitations of the RBAC model and its usage today:

Lack of expressiveness. The role-based implementation described above does not capture all the constraints of our desired policy. The role-based implementation allows doctors to access and modify any patient's record, rather than only their own patients. Similarly, the role-based implementation allows patients to access any other patient's record. One way to solve the problem is to give each user his or her own role, but that would remove the advantages of using roles altogether! Simply put, the RBAC model is not fine-grained enough to express common access control requirements.

As a result of this limitation, programmers may be forced to insert manual access checks that augment the ones provided by systems like Java EE. This manual process is error prone, and it is difficult to ensure that the inserted checks properly enforce the desired policy. Alternatively, a system may only enforce a coarse-grained access control policy but additionally maintain a $\log$ of accesses to allow system administrators to detect finer-grained violations a posteriori. ${ }^{1}$

Lack of static checking. The reliance solely on dynamic checks in today's RBAC-based systems leads to several problems. First, it is difficult for programmers to ensure that their code properly respects the access control policy. Programmers must manually keep track of what roles must be held when each function is invoked, which depends on the set of privileged operations that can potentially be reached during the function's execution. If a function is ever executed in the wrong environment, the only feedback will be a runtime role failure when a privileged operation is invoked, making the problem difficult to diagnose and fix.

Further, because of the cost of runtime role checks, the checks are often hoisted from the privileged operations themselves to the "entry points" of an application. For example, after user authentication, a single role check could be used to determine which web page to display (e.g., one for doctors and another for patients). However, in this case the programmer must manually ensure the sufficiency of this check for all potentially reachable privileged operations downstream, or else the intended access policy can be subverted.

In this paper we address both of these limitations of the traditional RBAC model and associated frameworks. First, we extend the RBAC model to support fine-grained policies like that of our medical records example above. The basic idea is to allow roles and privileged operations to be parameterized by a set of index values, which intuitively are used to distinguish users of the same role from one another. A privileged operation can only be invoked if both the appropriate role is held and the role's index values matches the operation's index value.

\footnotetext{
${ }^{1}$ This was the case in two recent security breaches in the news: unauthorized access to Britney Spears' medical records by employees at UCLA medical center and to Barack Obama's cell phone records by employees at Verizon Wireless.
} 
Our parameterized form of RBAC, which we call Object-sensitive RBAC (OR$\mathrm{BAC})$, has a natural interpretation and design in the context of an object-oriented language (Sect. 2). Traditional RBAC policies control access at the level of a class. For example, with Java EE a method getHistory in a Patient class can be declared to require the caller to hold the Patient role. In other words, a user with the Patient role can invoke the getHistory method on any instance of Patient. In contrast, ORBAC supports access control at the level of an individual object. For example, getHistory can now be declared to require the caller to hold the Patient $<$ this.patientId $>$ role, where the patient Id field of Patient stores a patient's unique identifier.

Second, we provide a type system that statically ensures that a program meets a specified ORBAC policy, providing early feedback on potential access control violations. We formalize our static checker for a core Java-like language (Sect. 3). Since types and roles are parameterized by program values (e.g., this. patient Id), our static checker is a form of dependent type system.

We have implemented our static type system for ORBAC as a pluggable type system for Java in the JavaCOP framework [2]. As with frameworks like Java EE, we leverage Java's annotation syntax to specify the role requirements on method calls, but the JavaCOP rules statically ensure the correctness and sufficiency of these annotations. We have augmented the OpenMRS medical records application [21] with a fine-grained access control policy using ORBAC and have used our JavaCOP checker to statically ensure the absence of authorization errors (Sect. 4).

\section{Object-sensitive RBAC}

We now overview Object-sensitive RBAC and its associated static type system through a simple medical records example in Java, comparing an implementation using standard RBAC in Java EE with one using ORBAC.

\subsection{Role-Based Access Control}

An RBAC policy can be described as a tuple $(U, R, P, P A, U A)$ consisting of a set of users $U$, a set of roles $R$, and a set of permissions $P$, together with relations $P A \subseteq P \times R$ giving permissions to roles and $U A \subseteq U \times R$ giving (sets of) roles to users [9]. An access of permission $p$ by user $u$ is safe if there exists a role $r \in R$ such that $(u, r) \in U A$ (user $u$ has role $r$ ) and $(p, r) \in P A$ (role $r$ has permission $p$ ).

Figure 1 shows how this model applies to a Patient class for which we wish to protect access. Our simplified class provides a factory method getPatient, which retrieves the specified patient from the database, and two instance methods: getHistory to return a history of the patient's visits and addPrescription to associate a new prescription with the patient.

We can group the users of our application into two groups: doctors and patients. In a typical medical records application, doctors can access the data of their patients and patients can access their own data (e.g., through a web self-service feature). In a standard RBAC model, we can represent these two groups with Doctor and Patient roles. Java 


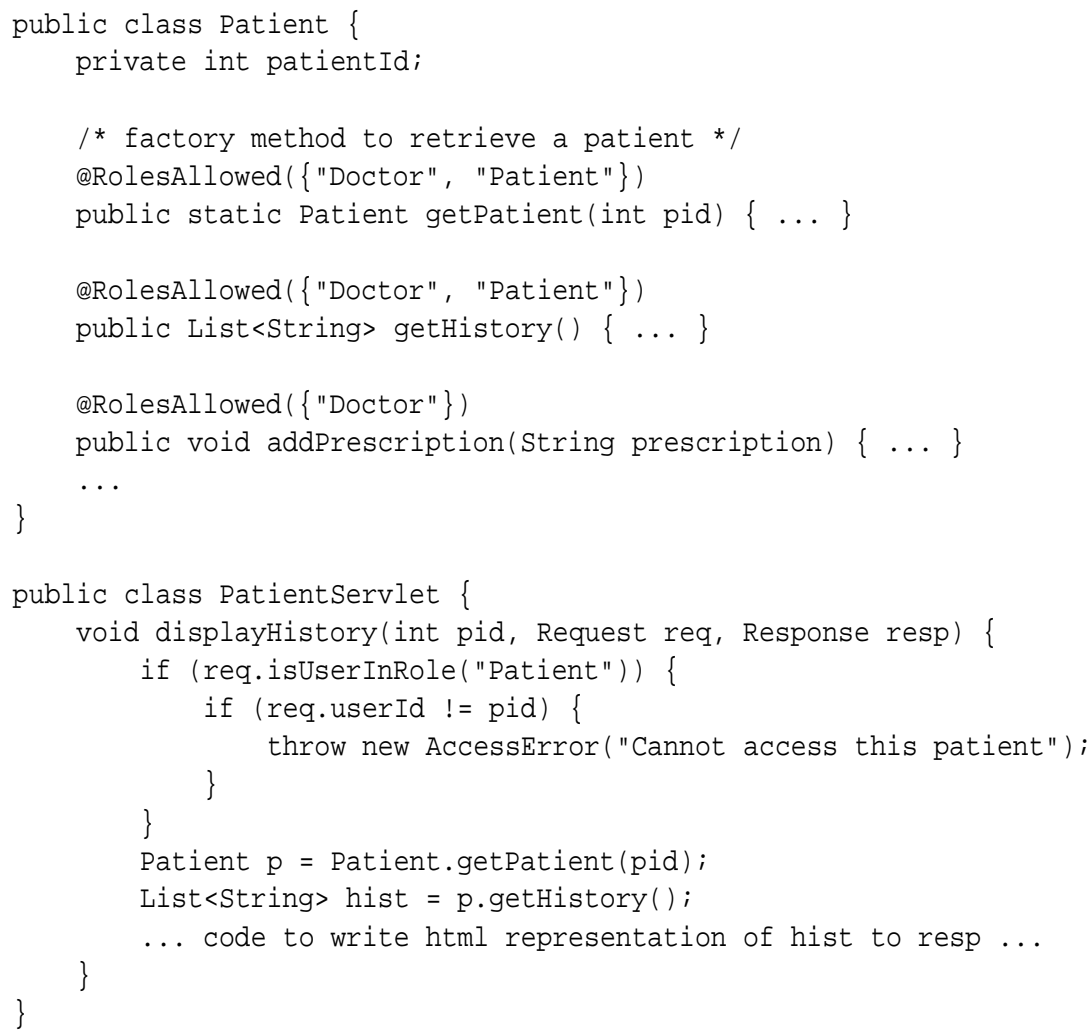

Fig. 1. Standard RBAC version of doctor-patient example

EE supports the specification of an RBAC policy through the @RolesAllowed annotation [16]. This annotation is placed on a method definition to indicate the set of roles that have permission to invoke the method. In Fig. 1 we have annotated the getPatient and getHistory methods to permit users with either the Doctor or Patient role to call these methods. On the other hand, the addPrescription method has been annotated to ensure that only doctors can add a prescription to a medical record.

The Java EE tools, and other application frameworks, enforce an RBAC policy dynamically by inserting runtime checks to verify that the user indeed has at least one of the specified roles when an annotated method is invoked. These checks are supported by standard infrastructure that performs user authentication and queries a database or configuration files to determine role membership.

For example, one might maintain a database of users and the roles granted to each user in an external LDAP server, where it can be managed by an administrator. The first time a user attempts to access a protected application resource (e.g., a web page), he is redirected to a login page. The user is authenticated by comparing his credentials 
against those stored in the LDAP server. The user's identity and roles are then stored in memory (e.g., in a session context) for use by dynamic access control checks.

Limitations of the RBAC model Consider the PatientServlet class of Fig. 1, which accesses a patient's medical record. The displayHistory method writes an HTML representation of the patient history to a response stream. To do this, it obtains a Patient object using Patient.getPatient and then calls its getHistory method. Due to the annotations on these methods, the Java EE framework will insert dynamic checks on these calls to ensure that the user has either the Doctor or Patient role.

Unfortunately, these checks are not sufficient to enforce the desired access control policy. For example, the checks allow any patient to access any other patient's medical record! Therefore, programmers must manually insert additional checks, as shown at the beginning of the displayHistory method. A similar check may also be necessary to ensure that a doctor only accesses the records of her own patients. These kinds of checks are very fragile and error-prone - one can easily forget or improperly implement the check on some code path that leads to an invocation of a protected method, resulting in a serious security vulnerability.

Another limitation of traditional RBAC frameworks like Java EE is the reliance solely on dynamic checks, which makes it difficult to statically ensure that application code in fact respects the access policy of a protected class. For example, the programmer must ensure that the displayHistory method is never invoked by a user who does not have either the Doctor or Patient roles. This requirement is completely implicit and can only be understood by examining the implementation of displayHistory (and in general the implementations of methods transitively called by displayHistory). If a program disobeys the requirement, the programmer will receive no warning about the error, which will instead result in a dynamic access check failure. Such dynamic errors can be difficult to diagnose and fix. Further, if the error is not expected by the calling code, it may result in very unfriendly behavior from the user's perspective (e.g., a Java uncaught exception).

\subsection{Object-sensitive RBAC}

ORBAC is a natural generalization of the formal model for RBAC defined above. With ORBAC, we define $U A \subseteq U \times R \times I$ to be a ternary relation, in which $U A(u, r, i)$ gives a user $u$ an indexed role $(r, i) \in R \times I$, where $I$ is a set of index values. Permissions are also indexed, and an access by user $u$ to the indexed permission $(p, i) \in P \times I$ is safe if there exists a role $r \in R$ such that $(u, r, i) \in U A$ (user $u$ has indexed role $(r, i))$ and $(p, r) \in P A$ (role $r$ has permission $p$ ).

In Fig. 2, we reimplement our example using an ORBAC policy. We use two roles: Patient and DoctorOf, both of which are parameterized by a patient identifier (a Java integer). A patient is given the Patient role for his own identifier, allowing him to access his own record but not those of other patients. A doctor is given a Doctor $O f$ role for each of her patients, allowing access to those patients but no others.

Conceptually, classes are now parameterized by a set of role indices, which are part of the class's static type, analogous with ordinary type parameters in Java. These role 


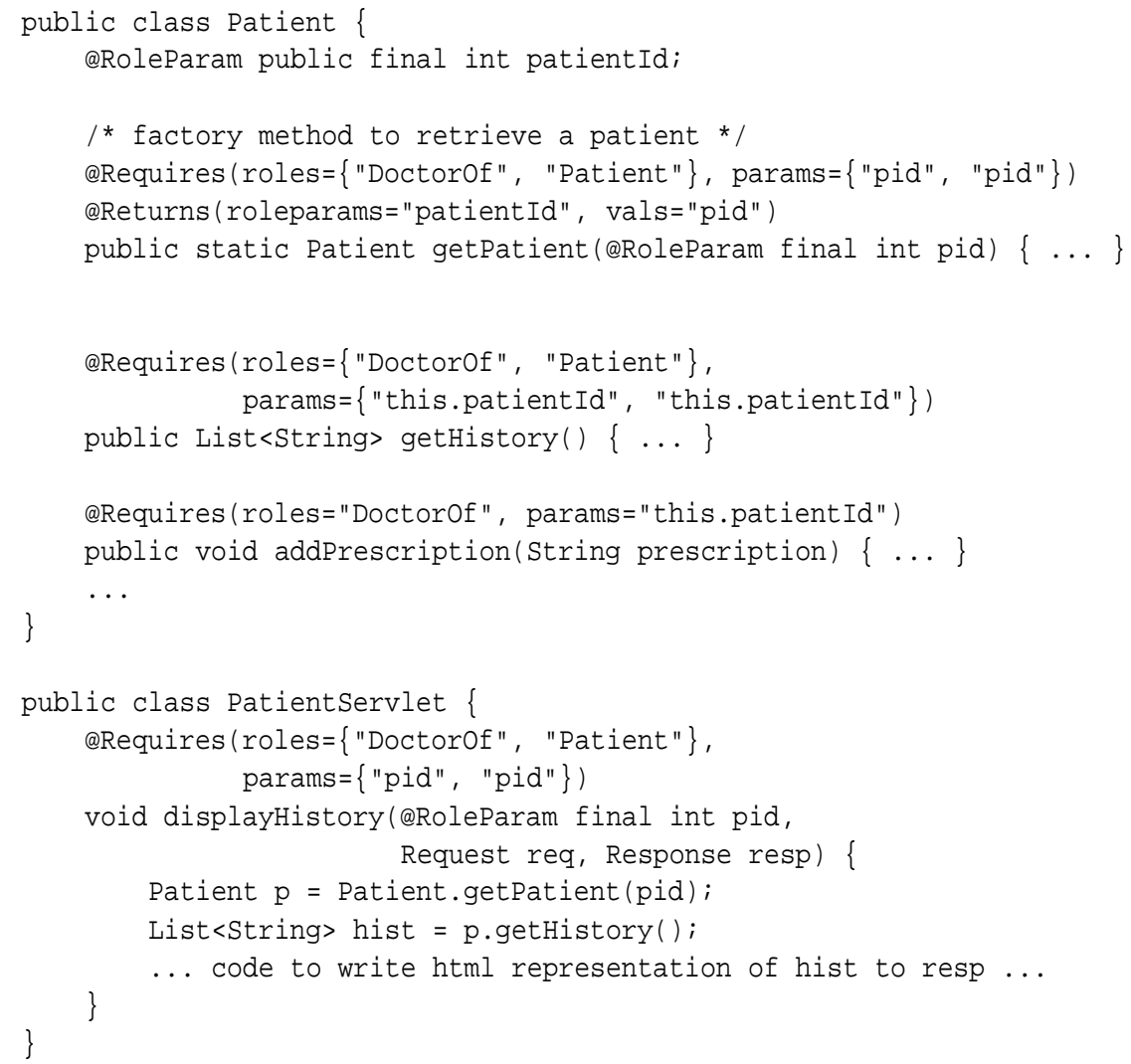

Fig. 2. ORBAC version of doctor-patient example

indices may then be used in role annotations within the class. While our formalism explicitly parameterizes classes in this way, as shown later, our implementation employs additional annotations to achieve the same effect without modifying Java's syntax. Class role parameters are modeled as public final fields of the class that are declared with the @RoleParam annotation. For example, the @RoleParam annotation on the patientId field of Patient indicates that this field will be used as an index in role annotations within the class. The @RoleParam annotation can also be used on final formal parameters to achieve the effect of method parameterization, as seen on the pid parameter of the getPatient method.

Our @Requires annotation is analogous to Java EE’s @RolesAllowed annotation, indicating the set of roles that have permission to invoke the annotated method. To stay within Java's metadata syntax we use two parallel arrays, roles and params, to specify the roles. For example, the @Requires annotation on getPatient in Fig. 2 allows only users with either the DoctorOf $\langle$ pid $>$ or Patient $<$ pid $>$ role to invoke the method, where pid is the patient identifier passed to the method. The @Requires annotations on the other methods are similar but they use the patientId field of the 
receiver as the role index to appropriately restrict access to that Patient object. Unlike the @RolesAllowed annotation, @Requires does not introduce a dynamic check. Instead, all calling code is statically checked to ensure at least one of the required roles is held.

The @Requires annotation is a form of method precondition for access control, while our eReturns annotation is a form of postcondition. For example, the @Returns annotation on getPatient asserts that the returned Patient object has a patientId role parameter field which is equal in value to the patient identifier passed to the method. Our static type system checks the body of the method to ensure the equality between the role parameters holds. The type system can then assume that this equality holds after a call to getPatient. In this way, we support modular typechecking for access control.

Resolving the limitations of the RBAC model The PatientServlet class of Fig.2 illustrates how ORBAC resolves the limitations identified earlier of the RBAC model. Unlike the version in Fig. 1, no manual access checks are required. These checks are now part of the access control policy and are reflected in the @Requires annotations on the methods of Patient. Therefore, it is easy for both humans and tools to reason about a program's access control policy just based on program annotations, without examining the bodies of methods.

Further, access control is now statically checked, providing early feedback on possible violations. The displayHistory method is annotated with @Requires, restricting the method to users of the DoctorOf $<$ pid $>$ and Patient $<$ pid $>$ roles. With this annotation, the method's body can be statically guaranteed to obey the access control policy of Patient. The call to getPatient satisfies that method's @Requires clause, so the call typechecks. The getPatient method's @Returns clause indicates that the returned patient object's patientId parameter is equal to pid, which then allows the call to getHistory to typecheck successfully.

Subtle errors are now caught statically rather than dynamically. For example, if the call to getPatient in displayHistory passed a patient identifier other than pid, the call would correctly fail to typecheck, since a patient could be accessing the record of a patient other than himself. Also, the annotation on displayHistory in turn allows its callers to be modularly checked at compile time, ensuring that they have the necessary roles for the eventual access to Patient.

Incorporating dynamic checks Our static type system makes explicit (via the @Requires annotation) the precondition that must be satisfied on entry to a method $m$ to ensure that the access control policies of all methods transitively called by $m$ will be obeyed. We insist that top-level methods (e.g., main for a standalone application or service for a servlet-based web application) have no @Requires annotation. That is, the application's external interface must have no precondition and thus can assume nothing about the roles that the current user holds. In order to allow an unprotected method to call a method protected by a @Requires annotation, our type system provides a flexible mechanism for interfacing with the program's authorization and authentication logic through the definition of role predicate methods. These methods are identified by the @RolePredicate annotation, which also indicates the role that the method tests for. Our static type system incorporates a simple form of flow sensitivity to ensure that 


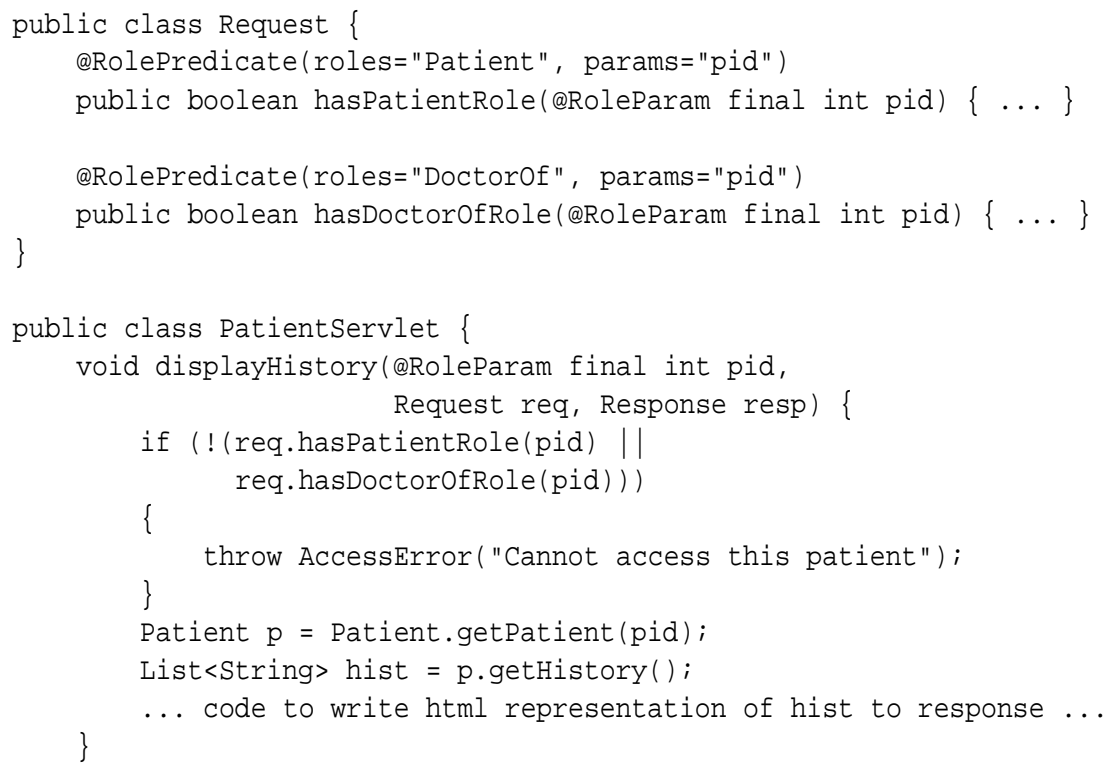

Fig. 3. Use of role predicate methods in displayHistory

method calls whose role requirements are not met by the current method's @Requires annotation occur only after appropriate dynamic checks succeed.

As a simple example, Fig. 3 contains a new version of PatientServlet's displayHistory method that performs the necessary role checks dynamically. The method no longer has a @Requires clause, but our static type system recognizes that the method is safe: the dynamic role checks ensure that the calls on the Patient class are only reached when the user has the appropriate Patient or DoctorOf role. Unlike the manual dynamic checks in the standard RBAC example shown earlier, these checks are statically ensured to be sufficient. Any errors in the dynamic checks in Fig. 3 (e.g., accidentally using a patient identifier other than pid) will be caught at compile time. Further, the dynamic checks can be placed as early as possible in the execution of an application without the risk that a check will be forgotten on some code path to a protected method.

The role predicate methods are treated as black boxes by our type system. They are free to consult a framework's security infrastructure or to implement authentication and authorization however the application designer sees fit. In fact, a particular predicate method could always return true and be used to achieve an effect similar to J2EE's @RunAs annotation, which allows components to be invoked with a security identity other than that of the currently authenticated user. In short, predicate methods provide a flexible mechanism for incorporating the runtime checks that are necessary to ascertain security credentials, and our type system ensures that their use is sufficient to satisfy declared method preconditions. 


$\begin{array}{lcll}\text { ClassDecl } & K & ::= & \text { class } C\langle\bar{r}\rangle\{\bar{T} f ; \bar{M}\} \\ \text { MethodDecl } & M & ::= & \langle\bar{r}\rangle T m(\bar{T} \bar{x}) \text { requires } \Phi\{e\} \\ \text { Exprs } & e & ::= & \begin{array}{l}x|e . f| e . m\langle\bar{\rho}\rangle(\bar{e}) \mid \text { new } T(\bar{e})|e \square e| \text { use } \Phi \text { in } e \\ \end{array} \\ \text { Vals } & v & ::= & \mid \text { pack } \rho, e \mid \text { unpack } e \text { as } r, x \text { in } e \\ \text { Types } & T & ::= & C\langle\bar{i}\rangle(\bar{\rho}) \mid \text { pack } i, v \\ \text { RoleContext } & \Phi & ::= & \text { propositional formula over atoms in } Q \\ \text { Roles } & Q & ::= & R\langle\rho\rangle \\ \text { Indices } & \rho & ::= & r \mid i \\ \text { IndexVarContext } & \Delta & ::= & \cdot \mid \Delta, r \\ \text { VariableContext } & \Gamma & ::= & \cdot \mid \Gamma, x: T\end{array}$

Fig. 4. Grammar for the ORBAC language and type system. Metavariable $C$ ranges over class names, $m$ over method names, $f$ over field names, $R$ over role names, $r$ and $q$ over index variables, $i$ and $j$ over index constants, and $x$ over program variables.

\section{Formal Semantics}

We have formalized the static and dynamic semantics of a small Java-like language in which ORBAC policies can be expressed and statically checked, and we have proven a type soundness theorem. Figure 4 shows the syntax of our language, a variant of Featherweight Java [14]. Our language models only the core features necessary to study the ORBAC model and its static type system formally. For this reason we have omitted inheritance, although our implementation handles it in the standard way, as described in Sect. 4.1.

In our Java implementation of ORBAC described in the previous section, index variables are specially designated fields and method parameters. In our formal language, we explicitly parameterize classes, methods, and roles using the syntax of Java generics. For greater expressiveness, we include a form of existential types to classify expressions whose role indices are not statically known. This models, for example, the situation in our Java implementation where a method's return type is parameterized by an index, but no information about this index's value is provided (e.g., via a @Returns annotation). Expressions of existential type are introduced in our core language by a pack expression and eliminated by an unpack expression, in the usual way [24]. Our core language includes a use expression for dynamically changing the set of held roles, which is a simplified form of the role predicate methods in our Java implementation. ${ }^{2}$ Finally, we include a non-deterministic choice construct $\left(e_{1} \square e_{2}\right)$ as a simple form of conditional.

Access protection is expressed in our Java implementation using a @Requires annotation indicating the set of roles that may invoke a method. This set can be viewed as a disjunctive predicate to be satisfied on entry to the method. We provide a more general mechanism in our formal language; methods include a requires clause which can specify an arbitrary propositional formula over roles as a precondition for invocation.

\footnotetext{
2 The use expression can be viewed as a role predicate method that always succeeds. The possibility of a predicate method returning false can be modeled by combining use with nondeterministic choice. For example, the expression (use $\Phi$ in $e_{1}$ ) $\square e_{2}$ models the situation where $e_{1}$ is executed if a dynamic check for predicate $\Phi$ succeeds, and otherwise $e_{2}$ is executed.
} 
$K$ ok

$$
\frac{\bar{r} \vdash \bar{T} \quad \bar{M} \text { ok in } C\langle\bar{r}\rangle}{\text { class } C\langle\bar{r}\rangle\{\bar{T} \bar{f} ; \bar{M}\} \text { ok }}
$$

$M$ ok in $T$

$$
\frac{\bar{r}, \bar{q} \vdash T \quad \bar{r}, \bar{q} \vdash \bar{T} \quad \bar{r}, \bar{q} \vdash \Phi \quad \Phi ; \bar{r}, \bar{q} ; \bar{x}: \bar{T}, \text { this : } C\langle\bar{r}\rangle \vdash e: T}{\langle\bar{q}\rangle T m(\bar{T} \bar{x}) \text { requires } \Phi\{e\} \text { ok in } C\langle\bar{r}\rangle}
$$

$\Phi ; \Delta ; \Gamma \vdash e: T$

$$
\begin{aligned}
& \Phi ; \Delta ; \Gamma \vdash x: \Gamma(x) \\
& \text { (T-VAR) } \\
& \frac{\Phi ; \Delta ; \Gamma \vdash e: T \quad \text { fields }(T)=\bar{T} \bar{f}}{\Phi ; \Delta ; \Gamma \vdash e \cdot f_{i}: T_{i}} \\
& \frac{\text { fields }(T)=\bar{T} \bar{f} \quad \Phi ; \Delta ; \Gamma \vdash \bar{e}: \bar{T} \quad \Delta \vdash T}{\Phi ; \Delta ; \Gamma \vdash \text { new } T(\bar{e}): T} \\
& \frac{\Phi ; \Delta ; \Gamma \vdash e_{1}: T \quad \Phi ; \Delta ; \Gamma \vdash e_{2}: T}{\Phi ; \Delta ; \Gamma \vdash e_{1} \square e_{2}: T} \\
& \frac{\Delta \vdash \rho \quad \Phi ; \Delta ; \Gamma \vdash e:[r \mapsto \rho] T}{\Phi ; \Delta ; \Gamma \vdash \operatorname{pack} \rho, e: \exists r . T} \\
& r \notin \Delta \quad \Gamma(x) \text { undefined } \\
& \frac{\Phi ; \Delta ; \Gamma \vdash e_{1}: \exists q . S \quad \Delta \vdash T \quad \Phi ; \Delta, r ; \Gamma, x:[q \mapsto r] S \vdash e_{2}: T}{\Phi ; \Delta ; \Gamma \vdash \text { unpack } e_{1} \text { as } r, x \text { in } e_{2}: T} \\
& \frac{\Delta \vdash \Phi^{\prime} \quad \Phi^{\prime} ; \Delta ; \Gamma \vdash e: T}{\Phi ; \Delta ; \Gamma \vdash \text { use } \Phi^{\prime} \text { in } e: T} \\
& \Phi ; \Delta ; \Gamma \vdash e: S \quad \Delta \vdash \bar{\rho} \quad \operatorname{msig}(S, m)=\langle\bar{r}\rangle \bar{T} \stackrel{\Phi^{\prime}}{\rightarrow} T \\
& \Phi ; \Delta ; \Gamma \vdash \bar{e}:[\bar{r} \mapsto \bar{\rho}] \bar{T} \quad \Phi \Rightarrow[\bar{r} \mapsto \bar{\rho}] \Phi^{\prime} \\
& \Phi ; \Delta ; \Gamma \vdash e . m\langle\bar{\rho}\rangle(\bar{e}):[\bar{r} \mapsto \bar{\rho}] T
\end{aligned}
$$

fields $(T)=\bar{T} \bar{f}$

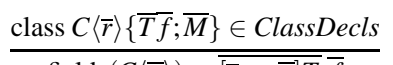

$\operatorname{msig}(T, m)=\langle\bar{r}\rangle \bar{T} \stackrel{\Phi}{\rightarrow} T$

$$
\frac{\text { class } C\langle\bar{r}\rangle\{\overline{T f} ; \bar{M}\} \in \text { ClassDecls } \quad\langle\bar{q}\rangle S m(\bar{S} \bar{x}) \text { requires } \Phi\{e\} \in \bar{M}}{\operatorname{msig}(C\langle\bar{\rho}\rangle, m)=\langle\bar{q}\rangle[\bar{r} \mapsto \bar{\rho}]]^{[\bar{r} \mapsto \bar{\rho}] \Phi}\left[{ }^{[} \mapsto \bar{\rho}\right] S}
$$

Fig. 5. Typing rules for our formal language. 
The typing rules for our formal language are shown in Fig. 5. Expressions are typechecked under three contexts: $\Phi$ is the role context represented as a propositional formula over roles, $\Delta$ keeps track of the index variables that are in scope, and $\Gamma$ is the usual free-variable typing context. The rules depend on a set of simple well-formedness judgments, which ensure that all referenced index variables are in scope. For example, $\Delta \vdash T$ in the premise of T-NEW ensures that the type being constructed does not refer to any undefined index variables.

The most interesting rule is T-INVK which includes a logical entailment check in the premise that guarantees that the current role context $\Phi$ satisfies the callee's requires precondition after appropriate substitution of actual indices for index parameters. Methods are typechecked modularly by rule M-OK which uses the $\Phi$ specified in a method's requires clause as the role context when checking the body.

Rules T-PACK and T-UNPACK are standard for existential type systems. The role variable $r$ in rule T-UNPACK is required to be fresh, which matches the intuition that existential types classify objects with unknown index values. An unpacked role variable $r$ can only be employed to satisfy role checks within a use statement that grants roles involving $r$. This is analogous to performing a dynamic role predicate check on an object with an unknown index in our Java implementation. Rules FIELDS and M-SIG only apply to class types, so an existential package must be unpacked before its fields and methods are accessed and values of existential type cannot be directly instantiated.

The dynamic semantics for our formal language is shown in Fig. 6. These evaluation rules perform role checks that model the dynamic checks on privileged operations used in most existing RBAC systems. Our type soundness result, however, establishes that such dynamic role checking is unnecessary for well-typed programs. Like the typing judgment, the evaluation judgment includes a role context. This context is used in rule E-INVK, which performs a dynamic entailment check that the current role context is sufficient to satisfy the method's declared precondition. Rule E-CONGRUENCE steps subexpressions according to the evaluation order established by the evaluation contexts, leaving the role context unchanged. Rule E-USE1 ignores the current role context and dynamically evaluates its subexpression under the specified context.

We have proven a type soundness theorem, which ensures that well-typed programs cannot fail dynamic role entailment checks. The theorem is proven using the standard progress and preservation style [30]. Full details are given in the accompanying technical report [10]; we provide statements of the key results here:

Lemma 1 (Progress) If $\Phi ; \cdot ; \cdot \vdash e: T$, then either $e$ is a value or there is an expression $e^{\prime}$ such that $\Phi^{\prime} \vdash e \longrightarrow e^{\prime}$ for any $\Phi^{\prime}$ where $\Phi^{\prime} \Rightarrow \Phi$.

Lemma 2 (Preservation) If $\Phi ; \Delta ; \Gamma \vdash e: T$ and $\Phi \vdash e \longrightarrow e^{\prime}$, then $\Phi ; \Delta ; \Gamma \vdash e^{\prime}: T$.

These lemmas imply a type soundness theorem as well as the key corollary about role checking:

Theorem 1 (Type Soundness) If $\Phi ; \cdot ; \cdot \vdash e: T$, then e will not get stuck when evaluated under any role context $\Phi^{\prime}$ such that $\Phi^{\prime} \Rightarrow \Phi$.

Corollary 1 (Dynamic Entailment Checks Unnecessary) Well-typed programs cannot fail dynamic role entailment checks. 
$\begin{aligned} \text { Evaluation Contexts } \quad E \quad::=\quad & {[]|E . f| E . m\langle\bar{\rho}\rangle(\bar{e}) \mid v \cdot m\langle\bar{\rho}\rangle(v, \ldots, E, e, \ldots, e) } \\ & \mid \text { new } T(v, \ldots, E, e, \ldots, e) \mid \text { pack } \rho, E \mid \text { unpack } E \text { as } r, x \text { in } e\end{aligned}$

$\Phi \vdash e \longrightarrow e$

$$
\begin{aligned}
& \frac{\Phi \vdash e \longrightarrow e^{\prime}}{\Phi \vdash E[e] \longrightarrow E\left[e^{\prime}\right]} \quad \text { (E-CONGRUENCE) } \\
& \frac{\text { fields }(T)=\bar{T} \bar{f}}{\Phi \vdash \operatorname{new} T(\bar{v}) \cdot f_{i} \longrightarrow v_{i}} \quad \text { (E-FIELD) } \\
& \frac{\operatorname{mbody}(T, m\langle\bar{\rho}\rangle)=(\bar{x}, e) \quad \operatorname{msig}(T, m)=\langle\bar{\sigma}\rangle \bar{S} \stackrel{\Phi^{\prime}}{\rightarrow} S \quad \Phi \Rightarrow[\bar{\sigma} \mapsto \bar{\rho}] \Phi^{\prime}}{\Phi \vdash \operatorname{new} T(\bar{v}) \cdot m\langle\bar{\rho}\rangle\left(\overline{v^{\prime}}\right) \longrightarrow\left[\bar{x} \mapsto \overline{v^{\prime}}\right][\text { this } \mapsto \text { new } T(\bar{v})] e} \text { (E-INVK) } \\
& \Phi \vdash \operatorname{unpack}(\operatorname{pack} i, v) \text { as } r, x \text { in } e \longrightarrow[x \mapsto v][r \mapsto i] e \quad \text { (E-UNPACK) } \\
& \Phi \vdash e_{1} \square e_{2} \longrightarrow e_{1} \quad \text { (E-CHOOSE1) } \\
& \Phi \vdash e_{1} \square e_{2} \longrightarrow e_{2} \quad \text { (E-CHOOSE2) } \\
& \frac{\Phi^{\prime} \vdash e \longrightarrow e^{\prime}}{\Phi \vdash \text { use } \Phi^{\prime} \text { in } e \longrightarrow \text { use } \Phi^{\prime} \text { in } e^{\prime}} \quad \text { (E-USE1) } \\
& \Phi \vdash \text { use } \Phi^{\prime} \text { in } v \longrightarrow v \quad \text { (E-USE2) }
\end{aligned}
$$

$\operatorname{mbody}(T, m\langle\bar{\rho}\rangle)=(\bar{x}, e)$

$$
\frac{\text { class } C\langle\bar{r}\rangle\{\bar{T} \bar{f} ; \bar{M}\} \in \text { ClassDecls } \quad\langle\bar{q}\rangle S m(\bar{S} \bar{x}) \text { requires } \Phi\{e\} \in \bar{M}}{\operatorname{mbody}(C\langle\bar{\rho}\rangle, m\langle\bar{\sigma}\rangle)=(\bar{x},[\bar{q} \mapsto \bar{\sigma}][\bar{r} \mapsto \bar{\rho}] e)} \quad \text { (M-BODY) }
$$

Fig. 6. Evaluation for our formal language. 


\section{Experience: The OpenMRS Case Study}

We implemented our ORBAC checker as an extension to Java in the JavaCOP pluggable types framework [2]. To evaluate our approach, we took OpenMRS [21], an existing open source medical records application, and retrofitted it to use an ORBAC policy to protect access to patient data. OpenMRS is implemented in Java using the Spring application framework [28], which is a commonly used alternative to Java EE. Spring provides several useful modules, including an inversion of control container, an aspectoriented programming framework, and integration with the Hibernate framework for persistence [13]. Spring's access control framework supports standard RBAC policies, which can be configured by an administrator.

\subsection{Implementation of ORBAC using JavaCOP}

Our checker implementation makes use of the annotations @Requires, @Returns, and @RolePredicate that were introduced in Sect. 2.2.

Several practical issues that are not modeled in the formalism are addressed in our implementation. Class inheritance is supported. The checker enforces the standard requirements on method overriding: an overriding method must have a compatible, possibly weaker precondition (@Requires annotation) and a compatible, possibly stronger postcondition (@Returns clause). Methods without a @Requires annotation are considered to have the precondition true, so they can be invoked in any context. Hence, methods that override such methods are required to not have a @Requires annotation.

While our formalism uses arbitrary propositional formulas for requires clauses, our Java implementation restricts @Requires and @RolePredicate annotations to be disjunctions of roles. This means that role contexts are formulas in conjunctive normal form (CNF); the @Requires clause of a method provides the first conjunct and dynamic role predicate checks add conjuncts to the context. This simplifies typechecking by allowing us to perform a series of subset checks rather than checking arbitrary logical implication.

We make use of JavaCOP's support for flow-sensitive reasoning [17] to implement the static updating of the role context based on role predicate method invocations. JavaCOP's flow framework properly handles Java's complex control flow, including exceptional control flow. As a result, our checker can statically validate the style of dynamic checks used in Fig. 3, as well as many other styles.

The implementation of the checker was fairly straightforward. It contains 174 lines of code in the declarative JavaCOP language and about 450 lines of Java code defining the flow analysis and some supporting functions and data structures.

\subsection{OpenMRS architecture}

The OpenMRS source contains over 160,000 lines of code, spread over 633 files, not including the frameworks and other infrastructure that it depends upon. Figure 7 shows a simplified UML diagram of some key patient-related classes defined by OpenMRS. Patients are represented by the Patient class. Each patient has a number of associated 

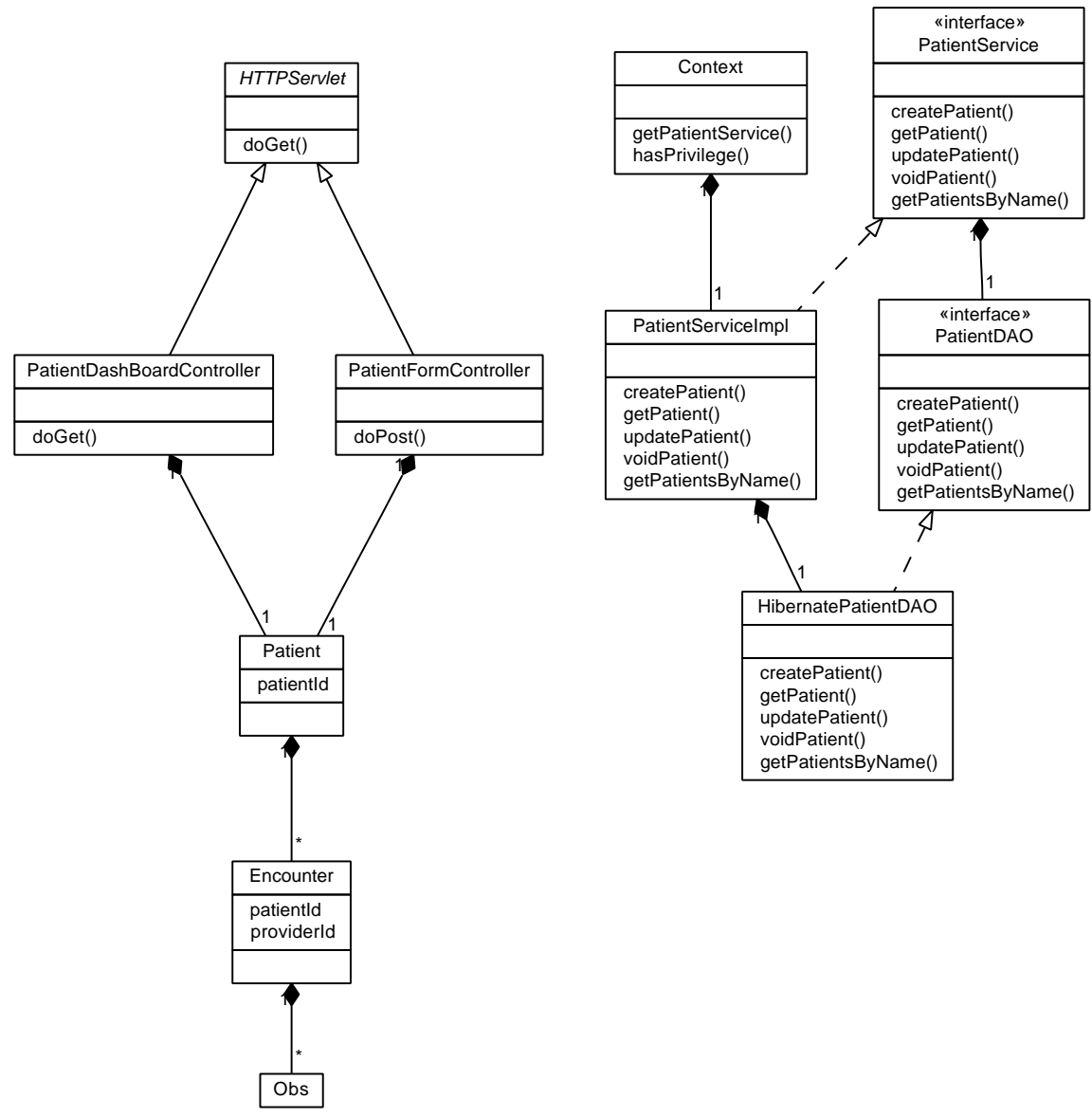

Fig. 7. Patient-related classes in OpenMRS

encounters, each representing a visit to the hospital or clinic. Each encounter may contain multiple observations (represented by the Obs class) which are used for recording test results and patient vitals.

The OpenMRS application interacts with the client via Java servlets. In Fig. 7, we show the two primary servlets for patients, PatientDashBoardController, which renders to HTML a summary of a patient's data, and PatientFormController, which accepts a new or updated patient and saves it to the database. These servlets obtain patient records from the database via classes implementing the PatientService interface, which defines methods for creating, querying, updating, and voiding patients (as well as many others not shown here). The implementation of PatientService is provided by PatientServiceImpl, which in turn uses a class implementing PatientDAO (DAO stands for "Data Access Object"). The implementation of PatientDAO is pro- 
vided by HibernatePatientDAO, which interacts with the Hibernate framework and isolates Hibernate-specific code.

The patient service implementation PatientServiceImpl is made available to servlets via the Context class. This class provides static methods for accessing global system state (e.g., mappings between "service" interfaces and their configured implementations) as well as state specific to a given user (e.g., a user id and permissions).

OpenMRS access control framework The implementation of RBAC in OpenMRS adds a level of indirection to the standard RBAC model: methods are protected by assigning required privileges through annotations in the code, roles are defined as mappings from role names to sets of privileges, and users are assigned sets of roles. The role-privilege mapping and the user-role mapping are maintained in the database, permitting them to be changed by an administrator at runtime.

Access policies are configurable in OpenMRS, but the limitations of the RBAC model make it impossible to configure a policy that permits access to a specific object while preventing access to other objects of the same class. In other words, only coarse-grained policies, which restrict access at the level of classes rather than objects, are supported. For example, in one reasonable policy within these restrictions, patients would have no access to the system at all and every healthcare provider would have read-write access to all patients.

Access control requirements are defined using method annotations representing the set of privileges needed to access the method. These annotations are converted to dynamic checks by Spring's aspect-oriented programming framework. For patient data, these annotations are made on the PatientService class. There are separate privileges defined for viewing, creating, updating, and deleting patients. The administrator must then assign these privileges to RBAC roles.

Each servlet in OpenMRS may (indirectly) invoke many dynamic privilege checks inserted by Spring. Unfortunately, there is no easy way to tell which privileges are required by a servlet. Changes to the implementation of a servlet may inadvertently change the set of privileges checked in a given situation, leading to runtime errors, which are displayed as an HTML rendering of a Java stack trace.

Privileges may be explicitly checked in the code by calling the hasPrivilege method on the Context class. These explicit checks are used in situations where authorization occurs in a conditionally executed block or where an implementation needs additional authorization requirements beyond those specified for an interface.

\subsection{An ORBAC policy for OpenMRS}

With ORBAC we were able to create a new fine-grained access control policy for patient objects, with three roles:

1. Users with the Supervisor role have read and write access to all patients. This role is unparameterized - it behaves as a standard RBAC role.

2. Users with the ProviderFor role (e.g., doctors) have read and write access to their patients, but not to other patients. This role is parameterized by the patient's id. 


\begin{tabular}{|l|l||l|l|l|}
\hline User & Assigned roles & $\begin{array}{l}\text { Patients allowed } \\
\text { read-only access }\end{array}$ & $\begin{array}{l}\text { Patients allowed } \\
\text { read-write access }\end{array}$ & $\begin{array}{l}\text { Patients denied } \\
\text { access }\end{array}$ \\
\hline \hline Alice & Supervisor & & $\begin{array}{l}\text { Britney, Carol, } \\
\text { Dave }\end{array}$ & \\
\hline Bob & ProviderFor $<$ Carol $>$ & & Carol & Britney, Dave \\
\hline Britney & Patient $<$ Britney $>$ & Britney & & Carol, Dave \\
\hline Carol & $\begin{array}{l}\text { Patient }<\text { Carol }>, \\
\text { ProviderFor }<\text { Britney }>\end{array}$ & Carol & Britney & Dave \\
\hline Dave & Patient $<$ Dave $>$ & Dave & & Britney, Carol \\
\hline
\end{tabular}

Fig. 8. Example of access rights for OpenMRS extended with ORBAC

3. Users with the Patient role have read access to their own patient record, but not to those of other patients. This role is parameterized by the patient's id.

We only changed the access policies for objects related to patients; other objects in the system are protected by OpenMRS's original RBAC policy.

Example 1. Figure 8 shows an example set of user-to-role assignments and the resulting access rights of these users. There are three patients in the system: Britney, Carol, and Dave. All three have a Patient role parameterized by their own id and can thus see, but not modify, their own patient records. Alice holds the unparameterized Supervisor role and has read-write access to the three patients. Bob is a provider for Carol, and thus has read-write access to her record, but no access to the other patients. Carol is both a provider for Britney and a patient herself. She does not have read-write access to her own record.

The mechanism for assigning the ProviderFor role turned out to be an interesting design consideration. The OpenMRS database schema and object model implement a one-to-many doctor-patient relationship, so one might consider using the presence of this relationship to grant ProviderFor status. However, in a real healthcare environment, multiple doctors and nurses might need to interact with a patient and thus see the patient's record. We chose to base the granting of the ProviderFor role on whether there is an encounter record associated with the patient and the provider. This can be determined by an SQL query against the Encounter table, the results of which can then be cached to speed up future checks.

The presumed workflow for granting access rights to a patient's data are as follows:

1. When a patient enters the clinic, a user with Supervisor access looks up the patient's record, or creates it if necessary.

2. The Supervisor selects a doctor to see the patient and then creates an encounter record referencing the patient and the doctor.

3. The doctor now has the ProviderFor role for this patient and can update the patient record.

Thus, all the providers who have participated in a patient's care can access the patient record. Other approaches to granting access rights to patient data are possible and enforceable with our pluggable type system. 
Implementing the ORBAC policy To implement our fine-grained access control policy in OpenMRS, we first made the patient Id field of the Patient class a role parameter via the $@ R o l e P a r a m$ annotation. We then replaced the original privilege annotations on the PatientService interface with @Requires annotations. For example, the declaration of the getPatient method is now:

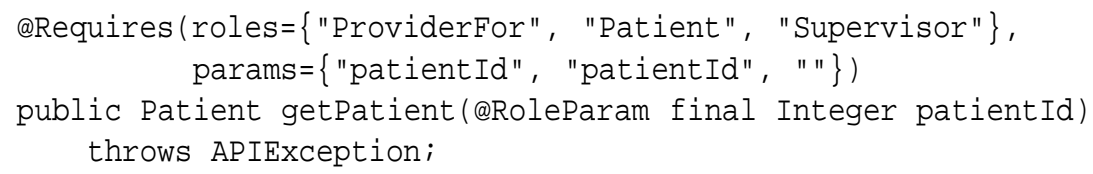

This method fetches the patient identified by patientId from the database. To call it, the caller must possess either the ProviderFor, Patient, or Supervisor roles. These first two roles are parameterized by the specific patientId, while Supervisor is unparameterized.

To provide dynamic role checks, we first created three new privileges in OpenMRS, corresponding to our three roles: ORBAC_PATIENT, ORBAC_PROVIDER, and ORBAC_SUPERVISOR. Each of these privileges has an associated OpenMRS role, which can then be assigned to users. We added role predicate methods for each of our ORBAC roles to the Context class. For example, the role predicate for the Patient role is defined as follows:

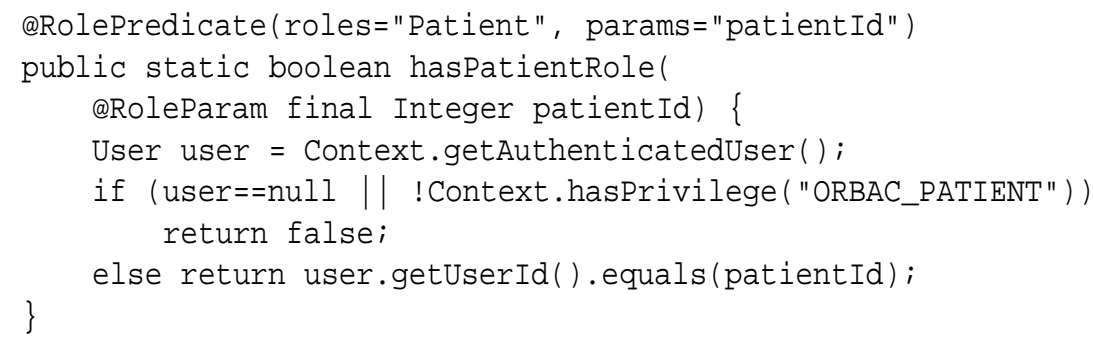

The method checks if the user has the OpenMRS privilege ORBAC_PATIENT and if so it compares the user's identifier to the specified patient identifier.

Checking the OpenMRS source code To ensure that the required roles for accessing patients were enforced, we ran our pluggable type system on the entire OpenMRS code base (a total of 633 Java files). The checking takes 11 seconds on a MacBook Pro with a $2.4 \mathrm{GHz}$ Intel Core 2 Duo processor and $2 \mathrm{~GB}$ of memory.

We used our type checker in an iterative manner in order to add necessary annotations and dynamic checks until all type errors were resolved. In general we used @Requires annotations on methods to remove static type errors. As mentioned in Sect. 2.2, we cannot place a @Requires annotation on the top-level methods in servlets through which all user requests must pass. This is the natural place to use predicate methods that perform dynamic security credential checks to satisfy the type checker.

In total, we made changes to 81 (13\%) of the files. A total of 298 @Requires annotations and 151 dynamic checks were added. Since the pluggable type system successfully checks the code, the dynamic role checks that occur within servlet code are guaranteed to be sufficient on all paths to the protected methods of PatientServiceImpl. 
The count of dynamic checks represents individual role predicate calls (hasPatientRole, hasProviderRole, or hasSupervisorRole). In many cases, these predicates are used together in a single if statement. In general, dynamic checks for patient reads use a disjunction of all three predicates, checks for patient writes use a disjunction of the provider and supervisor predicates, and checks for servlets that generate reports (which access many patients) use the supervisor predicate alone.

\subsection{Limitations and tradeoffs}

Final fields and role parameters In the ORBAC example of Sect. 2, role parameter fields are declared as final. Our type system requires that role parameters do not change. If role parameters can change, the type system becomes unsound, potentially allowing prohibited calls.

Unfortunately, Hibernate requires that persisted objects have default constructors and non-final id fields. These id fields are frequently the same fields used as role parameters (e.g., the patient Id of class Patient). To address this, we permit role parameter fields to be non-final but include checks in our pluggable type system to ensure that role parameter fields are not assigned outside of constructors. We also use the JavaCOP flow framework to ensure that every constructor initializes all role parameter fields.

ProviderFor vs. Provider roles In our case study, we chose to define for doctors a ProviderFor role which is parameterized by a patient id. This approach is straightforward and easily handles the case where a patient has multiple providers. However, it is problematic when representing collections. For example, the getPatientsByName method of PatientService takes a partial patient name and returns a Collection $<$ Patient $>$ of matching patients. The names of these patients are then displayed to the user, who can drill down to a specific patient record. We changed this method to return only those patients accessible to the user. Unfortunately, there is no way to represent the precise element type of this collection in our type system, since each patient has a different id. Therefore, we use a collection object with no role parameter. This lack of static validation cannot cause a security violation, but it does necessitate the use of dynamic role predicate checks in order to fetch the actual Patient object when the provider "drills down."

An alternative would be to instead use a Provider role, which is parameterized by the doctor's user id. Thus, the patients returned by getPatientsByName would all be parameterized by the same value, allowing easier representation in our type system.

This alternative approach is not without disadvantages. In the most obvious implementation of this policy, the Patient object would be parameterized by two fields: patientId and providerId. However, this does not work well if a patient can have multiple providers. One work-around is to change the getPatient method for PatientServiceImpl to populate the providerId with the current user's id, if the user is in the set of providers for the patient.

Access control for encounters and observations In our current implementation, accesses to objects logically contained within patients, such as encounters and observations, are not protected by eRequires annotations. In theory, this could lead to an 
unsoundness in the security policy, although, in practice, the OpenMRS navigation design prevents users from accessing these sub-objects without first accessing the parent Patient instance. To be sure there is no violation, we could add @Requires annotations to encounters and observations. Alternatively, we could use a form of object ownership [7] to verify that these objects are in fact logically contained within their associated patient objects.

\section{Related Work}

Role-based access control [9] has been used successfully in many systems and is now a NIST standard. Several approaches have been explored by researchers to extend declarative access control models like RBAC to represent and enforce instance-level policies. However, these approaches have employed only dynamic enforcement of such policies.

The emphasis in some prior work $[1,15,4]$ is on clarifying the formal semantics of a parameterized access control model. For example, Abdallah and Khayat [1] provide a set-theoretic semantics in a formal specification language, and Barth et al. [4] briefly mentions a parameterized role extension to a temporal logic for reasoning about privacy. We adapt a variant of these generalized RBAC models to an object-oriented language, provide a static type system for enforcing access control, and have implemented and validated the approach in Java.

The Resource Access Decision facility (RAD) [3] extends RBAC-based access control policies with access checks based on user relationships. Policies may be configured to require certain relationship predicates to be true when an activated role is used to access an object. For example, a rule might state that doctors can only access the records of patients to which they have an attending relationship. However, these relationship predicates are not defined in a declarative manner - a CORBA interface must be implemented in the application to evaluate each predicate. This precludes any use of a static analysis based on the relationships required by a policy.

The database community has also addressed the enforcement of instance-level access control policies (e.g., [12,26,20,22]). In particular, [12] extends RBAC with parameterized role templates, where the parameters of a template refer to database columns or constants and serve a similar function as our role parameters. Implementing fine-grained access control policies at the database level has two key advantages: one can define policies directly on the data to be protected and the filtering of records can be integrated with query optimization. However, database-level access control also has several disadvantages. First, it would be very difficult to statically determine the code paths in an application which lead to a given dynamically-generated SQL statement, which would be necessary to statically detect access violations. Second, developers may also want to enforce restrictions on function invocations in the application, which would require a separate mechanism from the database-level access control policies. Third, most modern application deployments store the mapping of users to roles in an external repository (e.g., an LDAP server). Information stored in such a repository might not be available to the database query engine.

Instance-level access control policies can also be defined using domain-specific languages. For example, the XAML standard [8] permits the definition of access policies 
for web services which reference data in individual request messages. Cassandra [6, 5] extends Datalog to express constraint rules referencing parameterized RBAC-style roles. These approaches are appropriate for enforcing access control between applications but are not so easily applied within an application. To (dynamically) enforce such policies within an application, one would need to map the entities referenced by the policy to actual object instances. In addition, the more expressive semantics of these policies would complicate static analysis.

We enforce access control policies through explicit dynamic and static checks added to the codebase through annotations. One could also write policies in a separate language outside the codebase and automatically insert them into the code at compile time or runtime (via bytecode manipulation). This approach has been explored [23], with policies expressed as access constraint rules - boolean expressions over an object and its relationships. Our ORBAC annotations could be translated to access constraint rules.

Our approach is orthogonal to Hierarchical RBAC [27], where a partial order is defined on roles. If a role $R_{1}$ is greater than a role $R_{2}$ in this hierarchy, then any user holding $R_{1}$ also holds the permissions associated with $R_{2}$. This hierarchy is statically defined and not dependent on individual object instances, so it still only supports coarse-grained policies. For example, if a Physician role dominates a Healthcare-Provider role in the hierarchy, assigning two users to Physician roles gives them the exact same permissions, which are a superset of the permissions granted to users assigned the HealthcareProvider role. One could extend our ORBAC model to support hierarchies by including a partial order on (parameterized) roles.

There has also been work on static analysis for RBAC systems. Closest to our work is that of Pistoia et al. on static analysis of security policies in Java EE [25]. They employ an interprocedural analysis to identify RBAC policies that are insufficient (i.e., can lead to runtime authorization failures), redundant (i.e., grants more roles than necessary), and subversive (i.e., allows bypassing access control requirements). Our static type system prevents the first and third of these errors, but for the more expressive ORBAC model. Using a type system as opposed to an interprocedural analysis allows us to provide modular guarantees about proper access control on each function in a scalable manner, at the expense of requiring user annotations.

Researchers have explored many forms of dependent type systems [18], whereby types depend on program values. The closest to our work is the notion of constrained types in the X10 programming language [19]. In X10, classes are explicitly parameterized by a set of properties, which are treated within the class as public final fields. Our design is similar but uses annotations to implicitly parameterize a class by a designated set of fields without modifying Java's syntax. Similarly, an X10 type has the form $C\{e\}$, where $C$ is a class name and $e$ is a constraint on the class's properties, while we use annotations to specify constraints. In our type system, these constraints are always simple equality constraints. The X10 compiler has built-in support for checking equality constraints, but it also allows users to plug in solvers for other constraints.

The static checking of roles in our type system has no analogue in X10's constrained types. This part of our type system is most closely related to type-and-effect systems [11], which statically track a set of computational effects. The computational effects we track are the privileged operations that a function may invoke, which de- 
termine the roles that are allowed to invoke the function. Roles are also similar to $c a$ pabilities [29], which are a dual to effects. However, roles are disjunctive rather than conjunctive: it is sufficient for an execution to hold any of a function's roles, while capability systems require all capabilities to be held to ensure proper execution.

\section{Conclusions}

We have presented the design, implementation, formalization, and practical validation of Object-sensitive RBAC (ORBAC), a generalization of the widely used RBAC model for access control. ORBAC allows different instances of the same class to be distinguished by a designated set of object properties. These properties can then be used to parameterize roles thereby supporting fine-grained access policies that are useful in common scenarios but hard to implement in traditional RBAC. We have implemented a novel static type system that employs forms of dependent types and flow sensitivity to provide sound yet precise reasoning about an application's adherence to an ORBAC policy. Our OpenMRS case study illustrates the practical utility of the ORBAC model and our type system in a realistic setting.

We have focused on a useful but restricted version of ORBAC. This model can be naturally extended to support a more expressive policy language. Our current JavaCopbased implementation could be enhanced to support role predicates as arbitrary propositional formulas as well as multiple parameters per role, both of which are in our formalization. Useful extensions to the type system presented here include the addition of

a partial order on roles, a richer constraint language for index values, and static tracking of the temporal order of privileged operations. Finally, we would like to investigate both local and global type inference of object-sensitive roles.

\section{References}

1. A. E. Abdallah and E. J. Khayat. A formal model for parameterized role-based access control. In T. Dimitrakos and F. Martinelli, editors, Formal Aspects in Security and Trust, pages 233-246. Springer, 2004.

2. C. Andreae, J. Noble, S. Markstrum, and T. Millstein. A framework for implementing pluggable type systems. In OOPSLA '06: Proceedings of the 21 st annual ACM SIGPLAN Conference on Object-Oriented Programming Systems, Languages, and Applications, pages 57-74. ACM Press, 2006.

3. J. Barkley, K. Beznosov, and J. Uppal. Supporting relationships in access control using role based access control. In RBAC '99: Proceedings of the fourth ACM workshop on Role-based access control, pages 55-65. ACM, 1999.

4. A. Barth, J. Mitchell, A. Datta, and S. Sundaram. Privacy and utility in business processes. In CSF'07, pages 279-294. IEEE Computer Society, 2007.

5. M. Becker. Information governance in nhs's npfit: A case for policy specification. International Journal of Medical Informatics (IJMI), 76(5-6), 2007.

6. M. Becker and P. Sewell. Cassandra: Distributed access control policies with tunable expressiveness. In POLICY '04, pages 159-168, 2004.

7. D. G. Clarke, J. M. Potter, and J. Noble. Ownership types for flexible alias protection. In Proceedings of the 13th ACM SIGPLAN conference on Object-oriented programming, systems, languages, and applications, pages 48-64. ACM Press, 1998. 
8. eXtensible Access Control Markup Language (XACML) Version 2.03. OASIS Standard, February 2005.

9. D. Ferraiolo and R. Kuhn. Role-based access control. In 15th National Computer Security Conference, 1992.

10. J. Fischer, D. Marino, R. Majumdar, and T. Millstein. Fine-grained access control with object-sensitive roles. Technical Report CSD-TR-090010, UCLA Comp Sci Dept, 2009.

11. D. K. Gifford and J. M. Lucassen. Integrating functional and imperative programming. In LFP '86: Proceedings of the 1986 ACM Conference on LISP and Functional Programming, pages 28-38. ACM Press, 1986.

12. L. Giuri and P. Iglio. Role templates for content-based access control. In RBAC '97: Proceedings of the second ACM workshop on Role-based access control, pages 153-159. ACM, 1997.

13. Hibernate home page. http://www.hibernate.org.

14. A. Igarashi, B. C. Pierce, and P. Wadler. Featherweight Java: a minimal core calculus for Java and GJ. ACM Transactions on Programming Languages and Systems, 23(3):396-450, May 2001.

15. T. Jaeger, T. Michailidis, and R. Rada. Access control in a virtual university. In WETICE '99: Proceedings of the 8th Workshop on Enabling Technologies on Infrastructure for Collaborative Enterprises, pages 135-140, Washington, DC, USA, 1999. IEEE Computer Society.

16. Java Platform, Enterprise Edition home page. http:// java.sun.com/ javaee.

17. S. Markstrum, D. Marino, M. Esquivel, and T. Millstein. Practical enforcement and testing of pluggable type systems. Technical Report CSD-TR-080013, UCLA Comp Sci Dept, 2008.

18. P. Martin-Löf. Constructive mathematics and computer programming. In Sixth International Congress for Logic, Methodology, and Philosophy of Science, pages 153-175, Amsterdam, 1982. North-Holland.

19. N. Nystrom, V. Saraswat, J. Palsberg, and C. Grothoff. Constrained types for object-oriented languages. In OOPSLA '08: Proceedings of the 23rd ACM SIGPLAN Conference on Object Oriented Programming Systems Languages and Applications, pages 457-474, New York, NY, USA, 2008. ACM.

20. L. Olson, C. Gunter, and P. Madhusudan. A formal framework for reflective database access control policies. In CCS '08: Proceedings of the 15th ACM conference on Computer and communications security, pages 289-298. ACM, 2008.

21. OpenMRS home page. http://openmrs.org.

22. Oracle $11^{g}$ Virtual Private Database, 2009. http://www.oracle.com/technology/deploy/security /database-security/virtual-private-database/index.html.

23. R. Pandey and B. Hashii. Providing fine-grained access control for Java programs. In ECOOP '99, volume LNCS 1628/1999, pages 668-692. Springer, 1999.

24. B. C. Pierce. Types and Programming Languages. The MIT Press, Cambridge, Massachusetts, 2002.

25. M. Pistoia, S. Fink, R. Flynn, and E. Yahav. When role models have flaws: Static validation of enterprise security policies. In ICSE '07, pages 478-488. IEEE, 2007.

26. S. Rizvi, A. Mendelzon, S. Sudarshan, and P. Roy. Extending query rewriting techniques for fine-grained access control. In SIGMOD '04: Proceedings of the 2004 ACM SIGMOD international conference on Management of data, pages 551-562. ACM, 2004.

27. R. Sandhu, E. Coyne, H. Feinstein, and C. Youman. Role-based access control models. IEEE Computer, 29(2):38-47, 1996.

28. Spring Application Framework home page. http://www.springsource.org.

29. D. Walker, K. Crary, and G. Morrisett. Typed memory management via static capabilities. ACM Trans. Program. Lang. Syst., 22(4):701-771, 2000.

30. A. K. Wright and M. Felleisen. A syntactic approach to type soundness. Information and Computation, 115(1):38-94, Nov. 1994. 